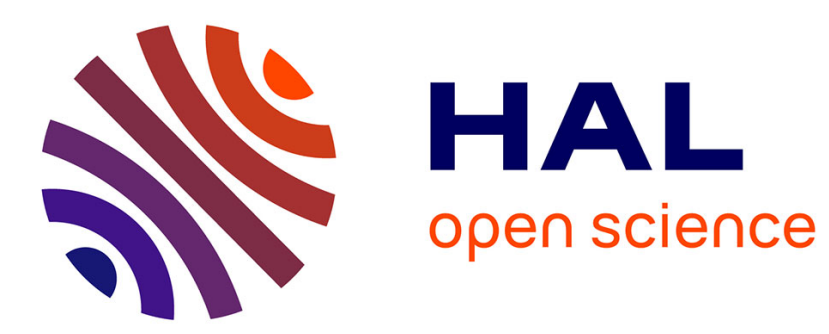

\title{
Laue diffraction lenses for astrophysics: From theory to experiments
}

\author{
H. Halloin
}

\section{To cite this version:}

H. Halloin. Laue diffraction lenses for astrophysics: From theory to experiments. Experimental Astronomy, 2006, 20, pp.171-184. 10.1007/s10686-006-9063-0 . in2p3-00130760

\section{HAL Id: in2p3-00130760 https://hal.in2p3.fr/in2p3-00130760}

Submitted on 13 Feb 2007

HAL is a multi-disciplinary open access archive for the deposit and dissemination of scientific research documents, whether they are published or not. The documents may come from teaching and research institutions in France or abroad, or from public or private research centers.
L'archive ouverte pluridisciplinaire HAL, est destinée au dépôt et à la diffusion de documents scientifiques de niveau recherche, publiés ou non, émanant des établissements d'enseignement et de recherche français ou étrangers, des laboratoires publics ou privés. 


\title{
Laue diffraction lenses for astrophysics : from theory to experiments
}

Received: date / Accepted: date

\begin{abstract}
Based on the laws of X-ray diffraction in crystals, Laue lenses offer a promising way to achieve the sensitivity and angular resolution leap required for the next generation of hard X-ray and gamma-ray telescopes.

The present paper describes the instrumental responses of Laue diffraction lenses designed for nuclear astrophysics. Different possible geometries are discussed, as well as the corresponding spectral and imaging capabilities. These theoretical predictions are then compared with Monte-Carlo simulations and experimental results (ground and stratospheric observations from the CLAIRE project).
\end{abstract}

Keywords Focusing optics · Gamma-ray astrophysics · Crystal diffraction

PACS 95.55.Ka $\cdot 61.50 . \mathrm{Ah} \cdot 61.10 . \mathrm{-i} \cdot 41.50 .+\mathrm{h}$

\section{Introduction}

Due to the very short wavelength of $\mathrm{X}$ and gamma rays, focusing instruments in high energy astrophysics has long been considered as impossible. Present telescopes in gamma-rays make use of direct shadowing (e.g. coded aperture telescopes such as INTEGRAL/SPI) or incoherent scattering (e.g. Compton telescopes such as CGRO/Comptel). Nevertheless, only a few years after the discovery of coherent scattering in crystal lattices by Friedrich, Knipping and Laue in 1912 [1], Gouy suggested an instrument focusing X-rays [2]. Following this method, Dardord in 1922 [3] and Fermi in 1923 [4] seem to have been

Hubert Halloin

CESR, 9, avenue du Colonel Roche, 31028 Toulouse, FRANCE

Present address: APC, 11, place Marcelin Berthelot, 75231 Paris Cedex 05, FRANCE Tel.: +33144271533

Fax: +33143546989

E-mail: hubert.halloin@apc.univ-paris7.fr 
the first to obtain images by mean of X-ray focusing. Though commonly used in crystallography, the application of Laue diffraction to high energy astrophysics is much more recent. One can refer to the work of Lindquist and Weber in 1968 [5], Smither in 1982 [6] and Lund in 1992 [7]. Recently, the first observation of an astrophysical source (the Crab nebula) has been performed during a stratospheric flight (the CLAIRE project, see the article by P. von Ballmoos et al. [8] in this volume and $[9,10])$. A spaceborne gammaray lens project, MAX, has also been proposed to the French Space Agency (see the corresponding article by N. Barriere [11] in this volume and $[12,13]$ ).

The theoretical basis of Laue diffraction lenses for astrophysics has already been given in another article in this volume ([14], hereafter cited as Paper 1). In the present article, the geometry and instrumental response of a Laue lens are more specifically studied. The expected instrumental response from X-ray diffraction theory is then compared with the results of ground and stratospheric observations.

\section{Design of a gamma-ray lens}

As presented in Paper 1, it is possible to coherently deviate hard X-rays and gamma-rays in crystals, using the Bragg relation :

$$
2 d \sin \theta=n \lambda,
$$

where $d$ is the planar spacing of the crystal, $\theta$ the incident angle, $\lambda$ the beam wavelength and $n$ an integer. Moreover, the Darwin model of mosaic crystals predicts efficiencies that are suitable for astrophysical purposes. These properties can be used to design a Laue diffraction lens.

Consider an X-ray beam impacting a crystal at Bragg angle $\theta_{B}$. After diffraction the beam direction is changed by $2 \theta_{B}$. With a ring of crystals, the diffracted beam can be focused on a single point. If appropriate d-spacing are used with different radii the same energy is diffracted from several rings. Alternatively, using the same diffracting plane at different radii allows the energy bandwidth of the lens to be increased. In this section, we will describe some properties and characteristics of a Laue lens.

\subsection{Choosing the diffraction material}

The choice of the diffracting material is crucial in the design of a Laue lens. It is a trade-off between diffraction efficiency, absorption and practicability of crystal growth. As mentioned in Paper $1, \S 3.1$, the atomic scattering factor tends to $\mathrm{Z}$ for small incident angles. So, as a first approximation, the diffraction efficiency of a crystal is linked to its electronic density (see fig. 1). A more precise analysis would nevertheless require consideration of the lattice geometry of the crystal (via the geometrical factor) and the absorption (which increases with $\mathrm{Z}$ and decreases with energy). For our purpose here, the electronic density is a reasonably good indicator of the "quality" of the material. 


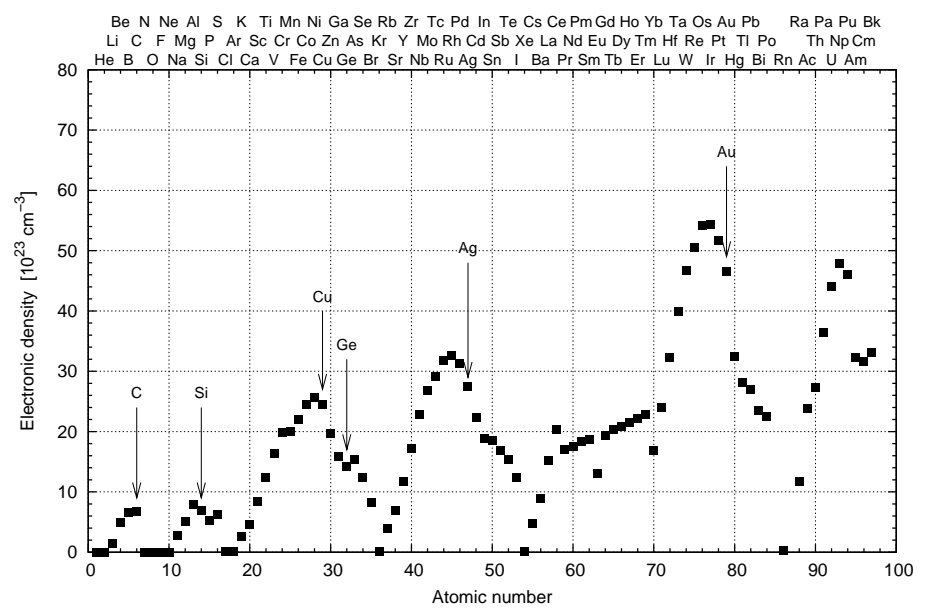

Fig. 1 Electronic densities of pure materials. Materials that have been considered for Laue lenses are indicated.

Among the "best" materials, at present only a few ones can be grown with the appropriate quality and mosaicity. Some of these candidates are indicated in fig 1. Carbon (actually HOPG : Highly Oriented Pyrolitic Graphite) and silicon are widely used and their growth process is now well controlled thanks to the development of the semi-conductor industry (especially for silicon). The diffraction efficiency of these crystals is nevertheless quite low above a few tens of keV.

Copper is a good diffraction material. While copper crystals with mosaicities at the level of a few arcminutes are "commonly" grown, until recently it has been quite difficult to get homogeneous copper crystals with a mosaicity of the order of the arcminute, as required for a gamma-ray lens above about $100 \mathrm{keV}$. Recent improvements in the growth process of copper crystals achieve mosaicities of 1 arcmin or less (see the article of P. Courtois et al. [15], in this volume).

Germanium (actually $\mathrm{Ge}_{1-x} \mathrm{Si}_{x}$ ) crystals were used to build the first gamma-ray lens for astrophysics (the CLAIRE project, see [8] in this volume). Due to the amount crystals that had to be produced $(\approx 1000)$, a good knowledge of the GeSi growing process and crystals parameters relevant for gamma-ray lenses now exists (see the article of N.V. Abrosimov in this volume [16] and [17]).

Silver and gold are also promising candidates (especially at high energy) and have the same crystal structure as copper and similar melting temperature, although no attempt has yet been made to grow mosaic silver or gold crystals for a Laue lens. 
2.2 Geometry of a Laue diffraction lens

Let us now consider a crystal, located at a radius $r$ from the optical axis of the lens, and a source at a distance $D$ (see fig. 2). $\mathrm{E}_{\infty}, \theta_{\infty}$ and $\mathrm{F}_{\infty}$ are the energy, Bragg angle and focal distance for a source on axis, at infinity. Similarly, E, $\theta$ and $\mathrm{F}$ refer to a source at a distance $D$ from the lens.

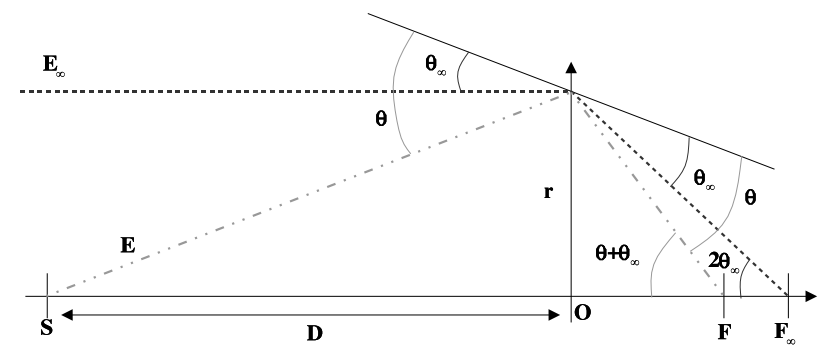

Fig. 2 Scheme of a crystal diffraction on a Laue lens

In the hard X- and gamma-ray regimes, the Bragg angles are small and the linearization of the Bragg relation leads to :

$$
\theta \approx \frac{h c}{2 d_{h k l} E} \approx 213.11\left(\frac{1 \AA}{d_{h k l}}\right)\left(\frac{100 \mathrm{keV}}{E}\right) \operatorname{arcmin}
$$

Additionally, if $D \gg r$, one has $\theta \approx \theta_{\infty}+\frac{r}{D}$ and so :

$$
\begin{aligned}
\frac{1}{E} & \approx \frac{1}{E_{\infty}}+\frac{2 d_{h k l}}{h c} \frac{r}{D} \\
\frac{100 \mathrm{keV}}{E} & \approx \frac{100 \mathrm{keV}}{E_{\infty}}+0.16128\left(\frac{d_{h k l}}{1 \AA}\right)\left(\frac{r}{10 \mathrm{~cm}}\right)\left(\frac{10 \mathrm{~m}}{D}\right)
\end{aligned}
$$

This equation specifies that it is equivalent (w.r.t the crystal planes orientation) to focus a beam of energy $E_{\infty}$ from infinity or one of energy $E$ at distance $D$. This property is very useful when tuning a Laue lens in laboratory, where the distance of the X-ray source is limited.

In addition we have, $F_{\infty} \approx \frac{r}{2 \theta_{\infty}}$ and $F \approx \frac{r}{\theta+\theta_{\infty}}$ and, consequently :

$$
\begin{aligned}
F_{\infty} & \approx \frac{r E_{\infty} d_{h k l}}{h c} \\
& \approx 0.80644\left(\frac{E_{\infty}}{100 \mathrm{keV}}\right)\left(\frac{r}{10 \mathrm{~cm}}\right)\left(\frac{d_{h k l}}{1 \AA}\right) \mathrm{m}
\end{aligned}
$$

and :

$$
\frac{1}{F}-\frac{1}{D} \approx \frac{1}{F_{\infty}}
$$

The latter equation is very similar to the equation of thin lenses (but with an associated dependence of the beam energy).

Eq. 4 allows to define two kinds of Laue lenses : 
- lenses with a few keV energy bandpass and consequently a relatively "short" (few meters) focal length

- those with a broad energy bandpass and therefore a long focal length (tens of meters).

Monochromatic Laue lenses In order to focus a single energy (from infinity) with crystals at different radii on the same focal point, the product $r \cdot d_{h k l}$ should be constant (from eq. 4). So, $r\left(\propto d_{h k l}^{-1}\right)$ can take only discrete values (e.g. $r \propto \sqrt{h^{2}+k^{2}+l^{2}}$ for cubic lattices).

From the Bragg relation, any angular deviation $\Delta \theta$ (either the mosaicity or source's depointing) leads to an energy offset $\Delta E \approx \frac{2 d_{h k l}}{h c} E_{0}^{2} \Delta \theta$. Since, in this configuration, all the ring diffracts the same nominal energy, any depointing greater than (roughly) the mosaicity causes a noticeable change in the energy response of the lens. The diffraction efficiency decreasing with $d_{h k l}^{-1}$, the contribution of the outer crystals rapidly becomes small and in practice the number of rings is limited.

The demonstration lens built for the CLAIRE project had to be flown on a stratospheric balloon and was consequently of this kind due to the requirement of a short focal length. This lens had a external diameter of about $40 \mathrm{~cm}, 8$ rings of germanium crystals ((111) to (440) planes), a nominal diffracted energy of $170 \mathrm{keV}$ at infinity and a focal length of $2.7 \mathrm{~m}$ (see [8] in this volume and references therein).

Broad band lenses In this case, the diffracted energy depends on the radius and the lens covers a wider energy bandpass. Actually, from one ring to another (with radii $R$ and $R+\Delta r, \Delta r \ll R$ ), the diffracted energies differ by $\Delta E \approx d_{h k l} E_{\infty}^{2} /\left(F_{\infty} h c\right) \Delta r$. On the other hand, the FWHM of the diffracted peak for a given ring is linked to the mosaicity $m$ of the crystal $: \Delta E \approx 2 m d_{h k l} E^{2} /(h c)$. Consequently, there is an energy overlap between two identical crystals at different radii only if $\Delta r<2 F_{\infty} m$.

Typically, the desired angular resolution of the lens leads to required mosaicities of the order of 1 arcmin, whereas, for the lens and detector to be on the same spacecraft, the focal length should not be greater than about $10 \mathrm{~m}$. With these values $\left(m=1 \operatorname{arcmin}\right.$ and $\left.F_{\infty}=10 \mathrm{~m}\right)$, the smoothness of the energy response requires $\Delta r \lesssim 6 \mathrm{~mm}$. In this case, concentric Laue rings would require small crystal tiles, with the accompanying difficulties in terms of cutting process and positioning.

Nevertheless, following an idea proposed by Lund [7], the tiles can be mounted according to an Archimedes spiral, the crystal radius varying linearly with its azimuth angle. These geometry ensures a very smooth energy distribution and is foreseen for the HAXTEL project (see the article from F. Frontera in this volume [18] and [19]).

For a longer focal length $(\sim 100 \mathrm{~m})$, requiring separated platforms, the mosaic energy spread is enough to realize a smooth energy response, even in case of concentric rings (which usually simplifies the lens design). The MAX mission (see the article from N. Barriere in this volume [11] and [13]) is of this kind. 


\section{Spectral and imaging response of a Laue lens}

A Laue diffraction lens is similar to a "classical" optical lens as far as the capability of concentrating an on-axis source is concerned. Nevertheless, the observation of an off-axis source induces aberrations, i.e. a modification of the diffracted spectrum, associated with a deformation of the point spread function (PSF).

\subsection{On-axis point source}

In order to quantify the Laue lens response to a point source, consider a single ring of crystals. This ring is assumed to have a mean radius $r$, focusing an energy $E_{\infty}$ from an on-axis point source at infinity, on a detector located at the focal distance $\left(F_{\infty}\right)$. The size of the focal spot is due to the superposition of the tiles footprints, convolved by the radial divergence of the beam (see Paper $1, \S 7.3$ ). Whereas the size of the crystals footprints does not depend on the detector distance, the "mosaic defocusing" (as introduced by N. Lund in this conference) increases with the focal length. This effect also induces a decrease of the instrument sensitivity, since the background level scales with the detection area.

If the detector is close to the lens, or the mosaicity small, only the projection of the footprints has to be considered. In this case, the focal spot intensity distribution, diffracted by a full ring, is well approximated considering that the intensity at radius $r$ on the detector is proportional to the mean value of the intensity diffracted by a single crystal at the same radius from its center (assuming a perfect alignment of all tiles). Mathematically, if $2 L \times 2 l$ is the size of the tiles (with $L>l$ ), then the radial intensity is proportional to :

$$
I(r)= \begin{cases}1 & \text { if } r \leq l \\ 1-\frac{2}{\pi} \arccos \left(\frac{l}{r}\right) & \text { if } l<r \leq L \\ 1-\frac{2}{\pi}\left(\arccos \left(\frac{l}{r}\right)+\arccos \left(\frac{L}{r}\right)\right) & \text { if } L<r \leq \sqrt{l^{2}+L^{2}} \\ 0 & \text { if } r>\sqrt{l^{2}+L^{2}}\end{cases}
$$

When the divergence of the beam cannot be neglected, the same procedure using the radial intensity from Paper 1, eq. 57 leads to :

$$
I(r)= \begin{cases}\frac{2}{\pi} \int_{0}^{\pi / 2} I_{\text {det }}(r \sin \theta) d \theta & \text { if } r \leq d_{\perp} \\ \frac{2}{\pi} \int_{\arccos \left(d_{\perp} / r\right)}^{\pi / 2} I_{\text {det }}(r \sin \theta) d \theta & \text { if } r>d_{\perp},\end{cases}
$$

where $d_{\perp}$ is half the size of the crystal, perpendicular to the radius. As a consequence of this equation, $I(r) \propto I_{\text {det }}(r) / r$ if $r \gg d_{\perp}$.

The effect of the mosaic defocusing has a major impact on the sensitivity. The area of the focal spot doesn't depend on the focal distance $F_{\infty}$, provided that the beam divergence (due to the mosaicity) is small w.r.t. the crystals' footprint, i.e. if $F_{\infty} \ll \Delta r_{c} / m$, where $\Delta r_{c}$ is the half the radial size of a crystal. Conversely, it scales as $F_{\infty}^{2}$ if $F_{\infty} \gg \Delta r_{c} / m$. Consequently, considering 
a position sensitive detector, the background noise variability is independent of $F_{\infty}$ for short focal length and increases as $F_{\infty}$ otherwise.

The collecting area being proportional to $F_{\infty}^{2}$, the lens sensitivity scales as $F_{\infty}^{2}$ for short focal lengths and increases "only" linearly if $F_{\infty} \gg \Delta r_{c} / m$ (i.e. like a coded aperture telescope). This property is the main limiting factor, along with the mass constraint, to the increase of the focal length (and hence the effective area).

\subsection{Off-axis point source}

Calculating the off-axis response of a Laue ring (with no radial extension) shows that the focal spot is no longer a circle but a ring with an azimuthal dependence of the energy. This effect is due to the "mirror effect" on the diffracting planes and the equivalence between angle and energy via the Bragg relation. Actually, considering a point source at infinity with a small off-axis angle $\epsilon$, the incident angle on the crystal located at the azimuth $\chi$ on the ring is $\psi=\epsilon \cos (\chi-\phi)$, where $\phi$ is the azimuth of the source. Due to the reflection on the crystal planes, the impact positions on the detector are located on a circle of radius $F_{\infty} \epsilon$, centered on the lens axis :

$$
\left\{\begin{array}{l}
x(\chi)=F_{\infty} \epsilon \cos (2 \chi-\phi) \\
y(\chi)=F_{\infty} \epsilon \sin (2 \chi-\phi)
\end{array}\right.
$$

$\chi$ varying from 0 to $2 \pi$, the image on the detector is the superposition of 2 circles with the same radii and centers.

In addition, the energy diffracted by the crystal at azimuth $\chi$ is shifted from the nominal "on-axis" energy $E$ by : $\Delta E \approx-\frac{2 d_{h k l}}{h c} E^{2} \epsilon \cos (\chi-\phi)$.

Finally, if the position on the impact circle is parametrized by $\alpha=2 \chi-\phi$ $(\alpha \in[0: 4 \pi])$, then :

$$
\Delta E(\alpha) \approx-\frac{2 d_{h k l}}{h c} E^{2} \epsilon \cos ((\alpha-\phi) / 2), \quad \alpha \in[0: 4 \pi]
$$

This formula demonstrates that for the same impact position (i.e. for $\alpha \in[0$ : $2 \pi]$ and $\alpha+2 \pi)$, two energies are diffracted : $E \cdot\left(1 \pm \frac{2 d_{h k l}}{h c} E \epsilon \cos ((\alpha-\phi) / 2)\right)$.

Moreover, for $\alpha=\phi+\pi[2 \pi]$ the beam divergence is tangential to the impact circle, whereas its direction is along the radius for $\alpha=\phi[2 \pi]$. Consequently, the impacts density and spread depends on the position on the impact circle. Actually, the maximum impact density is at the "geometrical" position (i.e at the intersection of the detector plane and the line of sight).

According to Paper 1, eq. 57 and simplifying the rectangular footprint by a Gaussian distribution, the beam divergence induces a spatial dispersion whose FWHM is roughly given by $\sqrt{\left(2 \Delta r_{c}\right)^{2}+\left(2 F_{\infty} m\right)^{2}}$. So, two point sources (with one on-axis) can be separated by the lens provided that the diameter of the impact circle (from the off-axis source) is greater than the diameter of the "on-axis" spot size. This means $2 F_{\infty} \epsilon \gtrsim \sqrt{\left(2 \Delta r_{c}\right)^{2}+\left(2 m F_{\infty}\right)^{2}}$. The angular resolution of the lens is then approximately :

$$
\epsilon_{\text {min }} \approx \sqrt{\left(\Delta r_{c} / F_{\infty}\right)^{2}+m^{2}}
$$


Consequently, the angular resolution of a Laue lens improves with the focal length, but is roughly equal to the mosaicity as soon as $F_{\infty} \gtrsim \Delta r_{c} / m$.

The energy response to an off-axis point source can be considered as a convolution product : the response for a perfect pointing convolved by the "off-axis" broadening, whose width is roughly $\Delta E_{\epsilon} \approx 2 \frac{2 d_{h k l}}{h c} E^{2} \epsilon$, as explained above. Thus, in case of a broad band Laue lens (i.e. with a long focal length), the energy response is only slightly affected, whereas the PSF is quite sensitive to the off-axis angle. On the contrary, the energy response of a narrow line lens (i.e. with a short focal length) is strongly dependent on $\epsilon$, but not the impacts distribution.

\subsection{Monte-Carlo simulations}

The instrumental response (spectral and imaging) has been simulated using a Monte-Carlo software, specifically developed for Laue lenses simulations.

Two lens configurations have been simulated :

- a broad band (0.2-2 MeV) lens with a focal length of $150 \mathrm{~m}$

- a narrow band (3keV @170 keV) lens with a focal length of $2.77 \mathrm{~m}$

The broad band Laue lens is made of concentric rings of germanium, copper and gold crystals. The rings radii vary from $0.3 \mathrm{~m}$ to $4.45 \mathrm{~m}$, corresponding to an energy range from $200 \mathrm{keV}$ to $2 \mathrm{MeV}$. In order to limit the mass cost and foreseeing a deployable mechanism, the outer crystals are mounted on petals, as shown in fig. 3. In this case, diffraction orders from 1 to 3 have been considered. Additional information about the simulation is listed in table 1.

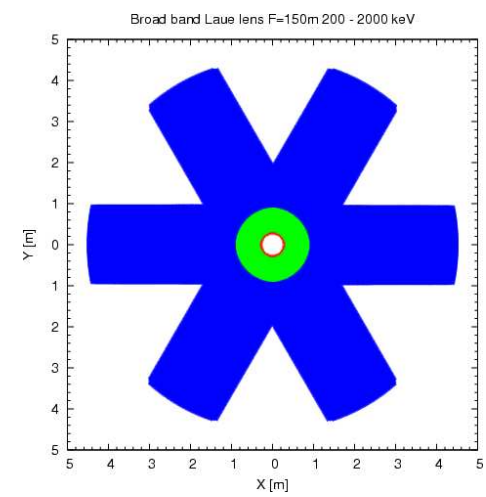

Fig. 3 Implantation of crystals for the MC simulation of a broad band Laue lens. From inner to outer rings : $\mathrm{Ge}_{111}, \mathrm{Au}_{111}$ and $\mathrm{Cu}_{111}$.

Comparisons are also made with a MC simulation of a narrow band Laue lens. For this simulation, the design of the CLAIRE lens has been used. It consists of $8 \mathrm{Ge}$ rings, focusing the same energy at infinity $(170 \mathrm{keV})$ with a focal length of $2.763 \mathrm{~m}$ (see table 2 ). 


\begin{tabular}{|l|c|}
\hline Parameter & Value \\
\hline Focal length & $150 \mathrm{~m}$ \\
Number of crystals & 92488 \\
Crystals size & $2 \times 2 \mathrm{~cm}^{2}$ \\
Crystal material (radius range) & $\mathrm{Ge}_{111}(0.31-0.39 \mathrm{~m})$ \\
& $\mathrm{Au}_{111}(0.41-0.97 \mathrm{~m})$ \\
& $\mathrm{Cu}_{111}(0.99-4.45 \mathrm{~m})$ \\
mosaicity & $30 "$ \\
crystals mass & $2131 \mathrm{~kg}$ \\
\hline
\end{tabular}

Table 1 Parameters of the broad band Laue lens used for Monte-Carlo simulations.

\begin{tabular}{|l|c|}
\hline Parameter & Value \\
\hline Focal length & $2.763 \mathrm{~m}$ \\
Number of crystals & 659 \\
Crystals size & $1 \times 1 \mathrm{~cm}^{2}$ (except rings 5 and 7 ) \\
& $1 \times 0.7 \mathrm{~cm}^{2}$ (rings 5 and 7) \\
Rings radii in mm & $61.7,100.8,118.2,142.6$, \\
& $156.18,174.64,188.15,201.67$ \\
mosaicity & 70 to 110 (depends on the ring) \\
Diffracting planes & $111,220,311,400$ \\
& $331,422,333,440$ \\
crystals mass & $2.1 \mathrm{~kg}$ \\
\hline
\end{tabular}

Table 2 Parameters of the narrow band Laue lens used for Monte-Carlo simulations.

The simulations have been performed for an on-axis source and for an off-axis angle of 3 arcminutes. The simulation results are illustrated on fig. 4 (broad band lens) and fig. 5 (narrow band lens). The simulated spectra are plotted in the upper part of the figures, above the density plot of the impacts on the detector, for the perfect pointing (right) and the off-axis source (left). Additionally, the spectral response of the broad band lens to an on-axis source has also been estimated using the analytical approximation of the total intensity diffracted by a crystal, as given in Paper 1, eq. 44. Compared to the MC simulation, this analytical approach reproduces quite well the spectral shape, though the "energy smoothing" due to the mosaicity is not taken into account. Using the analytical approximation has obviously the advantage of calculation time (a few seconds w.r.t. a few hours for the $\mathrm{MC}$ simulation) but, in the general case, it cannot easily reproduce the intensity map on the detector plane.

These MC simulations, based on the basic principles and equations of the Darwin model of crystals, confirm the spectral and imaging properties of a Laue lens, as described above. The energy response of a narrow band lens is very sensitive to the depointing, while the impact distribution is only slightly modified. On the contrary, the spectral shape of the broad band lens is almost unchanged between $200 \mathrm{keV}$ and $2 \mathrm{MeV}$, apart from the "sharp" features at 850 and $1800 \mathrm{keV}$ (which are smoothed). Conversely, the impact distribution becomes a ring, whose radius is about $13 \mathrm{~cm}\left(=150 \mathrm{~m} \times 3^{\prime}\right)$. 

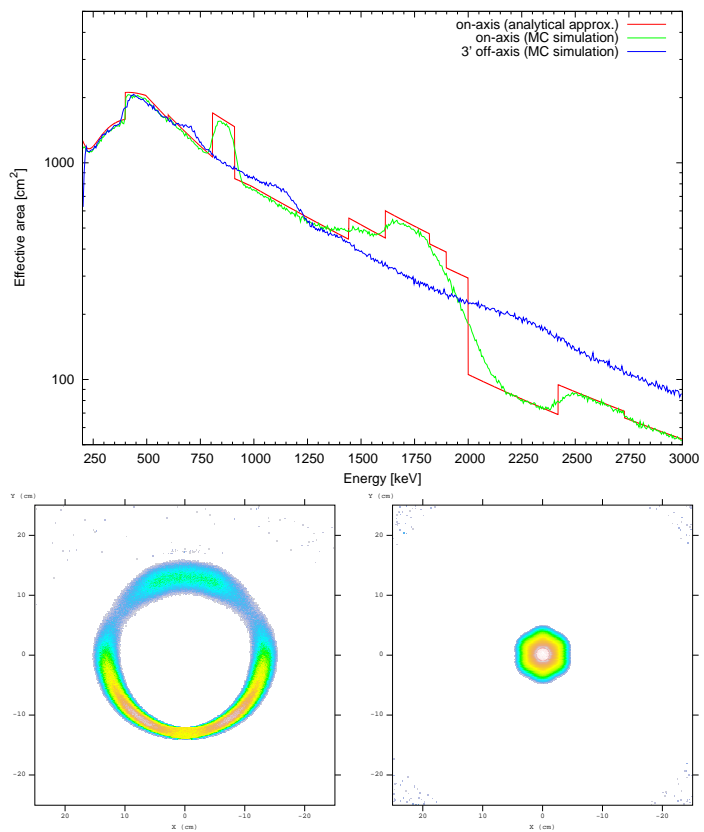

Fig. 4 MC simulation of a broad band Laue lens. Upper graph : diffracted spectra for an on-axis source (analytical approximation and Monte-Carlo simulation) and a 3 arcmin off-axis source. Lower graphs : Monte-Carlo simulations of impacts density on the focal plane (left : 3' off-axis source, right : on-axis).

\section{Theory vs. experimental results}

The CLAIRE project $[20,21,22,23,24]$ was intended to test the validity of the Laue lens concept on an astrophysical source. A narrow band Laue lens has been developed at the CESR (Toulouse, France) and tested on ground and during a stratospheric flight (observation of the Crab Nebula).

The characteristics (shape and flux) of spectra recorded during the lens tuning allowed the determination of the parameters of the crystals (mosaicity and mean length of the crystallites). These parameters were then used for the development of realistic numerical models (Monte-Carlo simulations), which can be compared with experiments in various conditions of pointing, source spectrum and distance, etc.

A detailed description of the CLAIRE project is given in P. von Ballmoos et al. ([8], this volume). Hereafter, we only quote the experimental results for comparison with theoretical expectations.

In order to estimate the diffraction efficiency of the lens, as well as its angular response, two experiments have been conducted on ground.

First, a radioactive source of ${ }^{57} \mathrm{Co}$ was observed with the lens. This source emits a line at $122.06 \mathrm{keV}$, corresponding to a distance of $14.07 \mathrm{~m}$ according to eq. 3. At this distance, the angular size of each crystal is $\sim 2.4$ arcmin (crystal height of $1 \mathrm{~cm})$ or $1.7 \operatorname{arcmin}(0.7 \mathrm{~cm})$. Since these values are larger than the mosaicity, only a small fraction of the crystal is diffracting, leading 

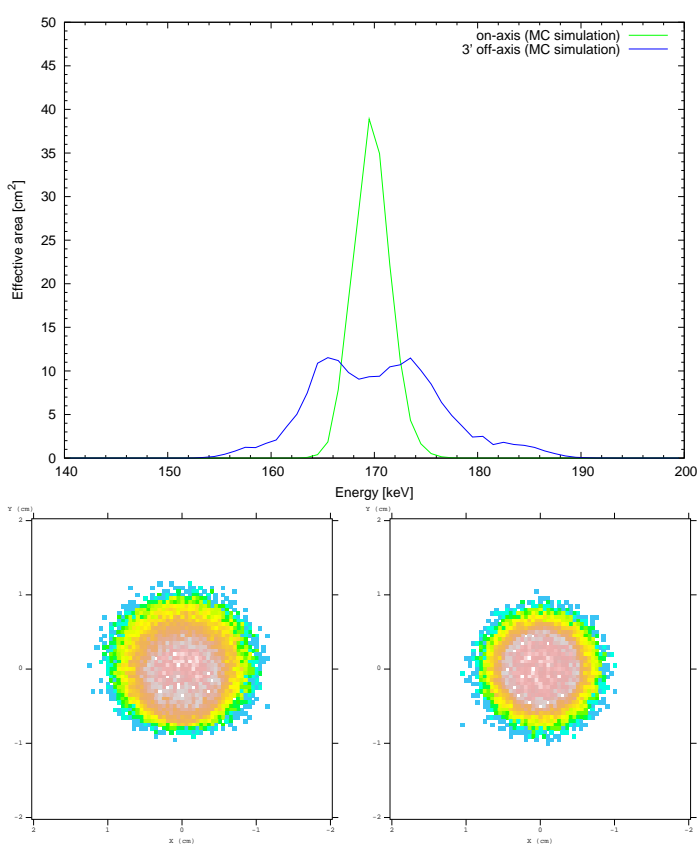

Fig. $5 \mathrm{MC}$ simulation of a narrow band Laue lens. See fig. 4 for a description of the figures.

to a diffraction efficiency (diffracted fraction of the incident radiation on the lens area) of $3.2^{ \pm 0.1 \%}$ (see Paper $1, \S 7.1$ ). The numerical simulations can then be used to correct this efficiency of a monochromatic, divergent beam into a polychromatic, parallel beam. Finally, taking into account the estimated uncertainties on the crystals' parameters, a semi-empirical value for the peak efficiency at $170 \mathrm{keV}$ (considering a diffracted peak of $3 \mathrm{keV}$ FWHM) can be set to $7.7^{ \pm 1} \%$.

Additional ground measurements with a source at $205 \mathrm{~m}$ were performed on an aerodrome in Figueras, on the Spanish Mediterranean coast [25]. This experiment led to a peak efficiency of $8.5^{ \pm 1} \%$, taking into account an estimation of systematic effects. Fig. 6 shows the energy response of the lens for various depointing angles (from 30 to 270 arcseconds). The curves are compared with the results of a Monte-Carlo simulation of these experiments. The shape and deformation of the energy response are well reproduced by the model. In that sense, this experiment validated the theoretical instrumental response of the lens.

On June 14 2001, CLAIRE was launched on a stratospheric balloon by the French Space Agency (CNES) from its site at Gap in the French Alps and landed near the Atlantic coast $500 \mathrm{~km}$ west of the launch site. After data analysis, the spectrum exhibits a significant excess of about 33 photons at $170 \mathrm{keV}$ with an exposure time of $1^{h} 12$. This result leads to a peak efficiency of $12.5^{ \pm 4_{-2}^{+0} \%}$, corrected for a perfect pointing and taking into account systematic effects. 


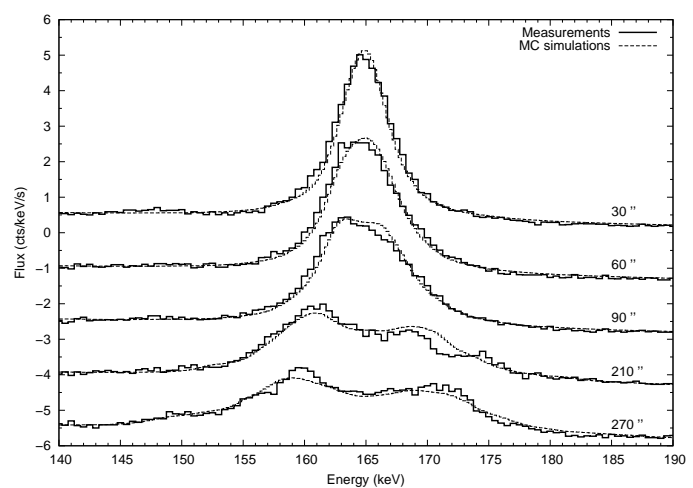

Fig. 6 Response of the CLAIRE lens during the long distance test, for various off-axis angles. The solid lines represent the measurements, the dashed lines are from an MC simulation. Successive plots have been shifted down for clarity.

\begin{tabular}{|c|c|c|c|}
\hline Experiment & Measured eff. $^{\mathrm{a}, \mathrm{b}}$. & Simulated eff. ${ }^{\mathrm{a}, \mathrm{c}}$ & Comments \\
\hline $\begin{array}{l}\text { Polychromatic } \\
\text { source at infinity }\end{array}$ & $12.5^{ \pm 4_{-2}^{+0}} \%$ & $8.88^{ \pm 0.02} \%$ & $\begin{array}{l}\text { Measurement derived from } \\
\text { the } 2001 \text { flight analysis }\end{array}$ \\
\hline $\begin{array}{l}\text { Polychromatic } \\
\text { source at } 205 \mathrm{~m}\end{array}$ & $8.5^{ \pm 0.5 \pm 0.5} \%$ & $8.53^{ \pm 0.02} \%$ & $\begin{array}{l}\text { Long distance test (see text } \\
\text { and }[25] \text { ) }\end{array}$ \\
\hline${ }^{57} \mathrm{Co}$ at $14 \mathrm{~m}$ & $3.17^{ \pm 0.02 \pm 0.1} \%$ & $3.668^{ \pm 0.004} \%$ & $\begin{array}{l}\text { Laboratory experiment with } \\
\text { a radioactive source }{ }^{\mathrm{d}}\end{array}$ \\
\hline \multicolumn{4}{|c|}{$\begin{array}{l}\text { a Peak efficiency assuming a peak FWHM of } 3 \mathrm{keV} \text { for polychromatic sources, diffracted } \\
\text { fraction of the incident flux on the lens for monochromatic sources. } \\
\mathrm{b} \text { Error bars include the statistical uncertainty (first figure) and an estimation of sys- } \\
\text { tematic effects (second figure). } \\
\text { c Error bars are only statistical. } \\
\text { d The low efficiency is due to the small diffracting volume for a monochromatic source } \\
\text { at finite distance (see text). Rescaling gives a peak efficiency of about } 7.7 \pm 1 \% \text { for a } \\
\text { polychromatic source at infinity. }\end{array}$} \\
\hline
\end{tabular}

Table 3 Comparison of experimental results and simulations

These experimental results are summarized and compared with numerical simulations in Table 3. Measurements and simulations are in good agreement and a value of $9^{ \pm 1} \%$ of peak efficiency for a polychromatic source at infinity is compatible with both experimental and simulated data.

Besides, the validity of the relationship between distance and diffracted energy (eq. 3) have been tested with various experiments using a continuum source :

- tuning data (distance of $14.16 \mathrm{~m}$ )

- source at $22.52 \mathrm{~m}$ with a partially tuned lens

- long distance test $(205 \mathrm{~m})$

- stratospheric flight (infinity...)

Fig. 7 represents the recorded spectra for these experiments (lower graph), compared with the theoretical relationship given by eq.3 (upper graph). The 


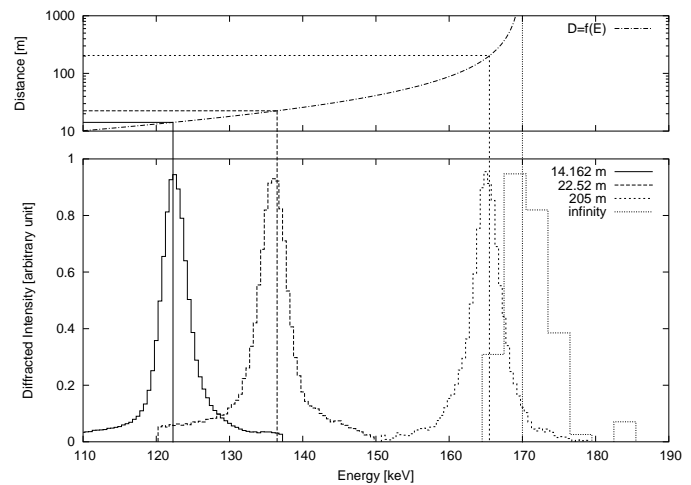

Fig. 7 Recorded spectra for continuum sources at various distances.Upper graph : theoretical positions of the peaks. Lower graph : results of the experiments.

position of the centroids are in very good agreement with theory, slight departures from theoretical values (less than $0.5 \mathrm{keV}$ ) being the consequence of the incident spectrum shape and/or the detector calibration drifts.

\section{Conclusion}

In the field of nuclear astrophysics, from a few tens of $\mathrm{keV}$ up to a few $\mathrm{MeV}$, future spaceborne instruments will have to perform a significant sensitivity and angular resolution leap. Present technologies, such as coded masks or Compton telescopes, have equivalent collecting and detecting area. Thus, their point source sensitivity seem to be "intrinsically" limited. Based on Xray diffraction in crystals, discovered almost 100 years ago, a Laue diffraction lens offer a way to overcome the present sensitivity dead-end, with also a unprecedented angular resolution in this energy range (typ. 1'). In the present paper, the main characteristics and design options of this kind of instruments have been described. Additionally, the expected instrumental responses are confirmed by Monte-Carlo simulations, as well as by experimental results. Once the behavior, expectations and limitations of Laue lenses for astrophysics are correctly understood, the next step is to focus on the best design for a future spaceborne gamma-ray lens...

\section{References}

1. P. K. W. Friedrich and M. Laue, Annalen der Physik 41, 971 (1913).

2. M. Gouy, Annales de Physique et de Chimie 5, 241 (1916).

3. R. Dardord, Journal de Physique et Le Radium 3, 218 (1922).

4. E. Fermi, Nuovo Cimento 25, 63 (1923).

5. T. Lindquist and W. R. Webber, Canadian Journal of Physics 46, S1103 (1968).

6. R. K. Smither, Review of Scientific Instruments 53, 131 (1982). 
7. N. Lund, Experimental Astronomy 2, 259 (1992).

8. P. von Ballmoos, H. Halloin, J. Evrard, G. Skinner, N. Abrosimov, J. M. Alvarez, B. Hamelin, M. Hernanz, P. Jean, J. Knödlseder, et al., this volume (2006).

9. H. Halloin, P. von Ballmoos, J. Evrard, G. K. Skinner, M. Hernanz, N. V. Abrosimov, P. Bastie, B. Hamelin, V. Lonjou, J. M. Alvarez, et al., in Optics for EUV, X-Ray, and Gamma-Ray Astronomy. Proceedings of the SPIE., edited by O. Citerrio and S. L. O'Dell (2004), vol. 5168, pp. 471-481.

10. H. Halloin, P. von Ballmoos, J. Evrard, G. Skinner, J. Alvarez, M. Hernanz, N. Abrosimov, P. Bastie, B.Hamelin, P. Jean, et al., in ESA SP-552: 5th INTEGRAL Workshop on the INTEGRAL Universe (2004), p. 739.

11. N. Barriere, P. von Ballmoos, H. Halloin, N. Abrosimov, J. Alvarez, K. Andersen, P. Bastie, S. Boggs, P. Courtois, T. Courvoisier, et al., this volume (2006).

12. P. von Ballmoos, H. Halloin, G. K. Skinner, R. K. Smither, J. Paul, N. V. Abrosimov, J. M. Alvarez, P. Astier, P. Bastie, D. Barret, et al., in Optics for EUV, X-Ray, and Gamma-Ray Astronomy. Proceedings of the SPIE., edited by O. Citerrio and S. L. O'Dell (2004), vol. 5168, pp. 482-491.

13. P. von Ballmoos, H. Halloin, J. Paul, N. Abrosimov, K. Andersen, P. Astier, S. Basa, D. Barret, P. Bastie, A.Bazzano, et al., in ESA SP552: 5th INTEGRAL Workshop on the INTEGRAL Universe (2004), pp. $747-753$.

14. H. Halloin and P. Bastie, this volume (2006).

15. P. Courtois, K. Andersen, and P. Bastie, this volume (2006).

16. N. Abrosimov, this volume (2006).

17. N. Abrosimov, H. R. n. V. K. A. Lüdge, D. Borrissova, H. Halloin, P. von Ballmoos, P. Bastie, B.Hamelin, and R. Smither, Journal of Crystal Growth 275, e495 (2005).

18. F. Frontera, this volume (2006).

19. A. Pisa, F. Frontera, P. De Chiara, G. Loffredo, D. Pellicciotta, V. Carassiti, F. Evangelisti, K. Andersen, P. Courtois, B. Hamelin, et al., in $O p$ tics for EUV, X-Ray, and Gamma-Ray Astronomy II. Proceedings of the SPIE., edited by O. Citerio and S. L. O'Dell (2005), vol. 5900, pp. 350-359.

20. J. E. Naya, P. von Ballmoos, R. K. Smither, M. Faiz, P. B. Fernandez, T. Graber, F. Albernhe, and G. Vedrenne, Nuclear Instruments and Methods in Physics Research Section A 373, 159 (1996).

21. A. Kohnle, R. Smither, T. Graber, P. von Ballmoos, P. Laporte, and J.F. Olive, Nuclear Instruments and Methods in Physics Research Section A 408, 553 (1998).

22. P. Laporte, N. V. Abrosimov, P. Bastie, B. Cordier, G. Di Cocco, J. Evrard, P. Laurent, P. Paltani, G. K. Skinner, R. K. Smither, et al., Nuclear Instruments and Methods in Physics Research Section A 442, 438 (2000).

23. P. von Ballmoos, J. Evrard, G. K. Skinner, N. Abrosimov, P. Bastie, G. Di Cocco, M. George, H. Halloin, B. Hamelin, P. Jean, et al., in 
ESA SP-459: Proceedings of the $4^{\text {th }}$ INTEGRAL Workshop 'Exploring the Gamma-Ray Universe' (2001), pp. 649-652.

24. P. von Ballmoos, Web page of the CLAIRE Project, http://www.cesr. $\mathrm{fr} /{ }^{\sim} \mathrm{pvb} / \mathrm{Claire} /$ index.html (2003).

25. J. Alvarez, H. Halloin, M. Hernanz, P. von Ballmoos, P. Jean, G. Skinner, N. Abrosimov, R. K. Smither, and G. Vedrenne, in ESA SP-552: 5th INTEGRAL Workshop on the INTEGRAL Universe (2004), pp. 757-+. 